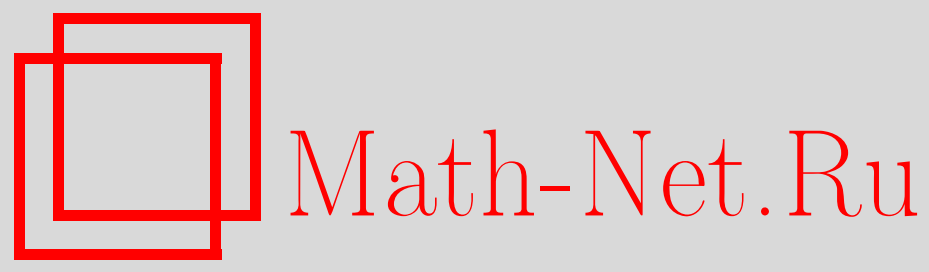

О. И. Мохов, Е. В. Ферапонтов, Уравнения ассоциативности двумерной топологической теории поля как интегрируемые гамильтоновы недиагонализуемые системы гидродинамического типа, Функи. анализ и его прил., 1996, том 30, выпуск $3,62-72$

DOI: https://doi.org/10.4213/faa538

Использование Общероссийского математического портала MathNet.Ru подразумевает, что вы прочитали и согласны с пользовательским соглашением

http://www . mathnet.ru/rus/agreement

Параметры загрузки:

IP : 54.224 .60 .19

26 апреля 2023 г., 14:48:21

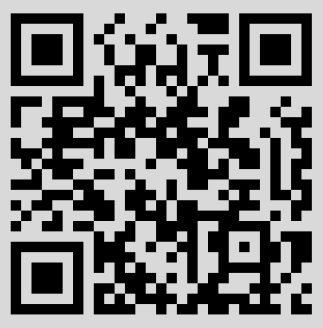




\title{
Уравнения ассоциативности двумерной топологической теории поля как интегрируемые гамильтоновы недиагонализуемые системы гидродинамического типа
}

\author{
(c) 1996. О. И. Мохов, Е. В. ФЕРАПонтов ${ }^{1}$
}

\section{$\S 1$. Введение}

Рассмотрим функцию $n$ переменных $F\left(t^{1}, \ldots, t^{n}\right)$, удовлетворяющую следующим двум условиям:

1. Maтрuиa

$$
\eta_{\alpha \beta}=\frac{\partial^{3} F}{\partial t^{1} \partial t^{\alpha} \partial t^{\beta}} \quad(\alpha, \beta=1, \ldots, n)
$$

является постоянной и невырожденной.

Отметим, что матрица $\eta_{\alpha \beta}$ полностью определяет зависимость функции $F$ от выделенной переменной $t^{1}$ (с точностью до квадратичных полиномов по $\left.t^{1}, \ldots, t^{n}\right)$.

2. Функиии

$$
c_{\beta \gamma}^{\alpha}(t)=\eta^{\alpha \mu} \frac{\partial^{3} F}{\partial t^{\mu} \partial t^{\beta} \partial t^{\gamma}} \quad\left(\text { здеcb } \eta^{\alpha \mu} \eta_{\mu \beta}=\delta_{\beta}^{\alpha}\right)
$$

для всех $t=\left(t^{1}, \ldots, t^{n}\right)$ лвляются структурными константами ассоциативной алеебры $A(t)$ в $n$-мерном пространстве с базисом $e_{1}, \ldots, e_{n}$ и умножением

$$
e_{\beta} \circ e_{\gamma}=c_{\beta \gamma}^{\alpha}(t) e_{\alpha} .
$$

Условия 1 и 2 налагают на функцию $F$ сложную переопределенную систему нелинейных уравнений в частных производных третьего порядка, известную в двумерной топологической теории поля под названием уравнений ассоциативности или системы Виттена-Дийкграафа-Г. Верлинде-Э. Верлинде (см. [10-13]; необходимые физические мотивировки и теорию интегрируемости уравнений ассоциативности можно найти в обзоре Дубровина [1]).

Для $n=3$ Дубровиным были рассмотрены два существенно различных вида зависимости функции $F$ от выделенной переменной $t^{1}$ :

$$
F=\frac{1}{2}\left(t^{1}\right)^{2} t^{3}+\frac{1}{2} t^{1}\left(t^{2}\right)^{2}+f\left(t^{2}, t^{3}\right) \quad \text { и } \quad F=\frac{1}{6}\left(t^{1}\right)^{3}+t^{1} t^{2} t^{3}+f\left(t^{2}, t^{3}\right) .
$$

\footnotetext{
${ }^{1}$ Работа выполнена при частичной финансовой поддержке Международного научного фонда Дж. Сороса, грант No. RKR000, Российского фонда фундаментальных исследований, гранты №94-01-01478 (О. И. М.) и №93-011-168 (Е. В. Ф.), и INTAS, грант No. 93-0166 (О. И. М.).
} 
Уравнения ассоциативности сводятся при этом к следующим двум нелинейным уравнениям третьего порядка на функцию двух независимых переменных $f=$ $f(x, t)$ (всюду далее $\left.x=t^{2}, t=t^{3}\right)$ :

$$
f_{t t t}=f_{x x t}^{2}-f_{x x x} f_{x t t}
$$

и

$$
f_{x x x} f_{t t t}-f_{x x t} f_{x t t}=1
$$

соответственно.

Введем, следуя [2, 3], переменные

$$
a=f_{x x x}, \quad b=f_{x x t}, \quad c=f_{x t t} .
$$

В новых переменных уравнения (1.1) и (1.2), как показано в [2, 3], приобретают вид систем гидродинамического типа порядка $3 \times 3$ :

и

$$
\left\{\begin{array}{l}
a_{t}=b_{x}, \\
b_{t}=c_{x}, \\
c_{t}=\left(b^{2}-a c\right)_{x}
\end{array}\right.
$$

$$
\left\{\begin{array}{l}
a_{t}=b_{x}, \\
b_{t}=c_{x}, \\
c_{t}=((1+b c) / a)_{x},
\end{array}\right.
$$

соответственно.

Для уравнений в частных производных второго порядка (типа Монжа-Ампера) аналогичное преобразование всегда приводит к двухкомпонентным системам гидродинамического типа, которые, как известно, линеаризуются методом годографа. В частности, в [17] показано, что уравнение Монжа-Ампера

$$
u_{t t} u_{x x}-u_{x t}^{2}=-1
$$

преобразованием

$$
U=\frac{u_{x t}}{u_{x x}}, \quad V=u_{x x}
$$

сводится к интегрируемой системе уравнений газа Чаплыгина:

$$
\left\{\begin{array}{l}
U_{t}=U U_{x}+V^{-3} V_{x}, \\
V_{t}=V U_{x}+U V_{x} .
\end{array}\right.
$$

Напомним, что системами гидродинамического типа называют системы квазилинейных уравнений вида

$$
u_{t}^{i}=v_{j}^{i}(u) u_{x}^{j} .
$$

Основным преимуществом записи уравнений ассоциативности в виде (1.3), (1.4) является наличие эффективной и хорошо разработанной теории интегрируемости систем гидродинамического типа - см., например, обзоры Царева [4], Дубровина и Новикова [5], а также работы [6-9], посвященные недиагонализуемым системам гидродинамического типа. В дальнейшем мы всюду рассматриваем 
строго гиперболические системы, т. е. такие системы, для которых собственные числа матрицы $v_{j}^{i}$ являются вещественными и различными. Так, обе исследуемые системы (1.3) и (1.4) являются строго гиперболическими.

В $\S 2$ устанавливается гамильтоновость системы (1.3). Для этой системы найдена локальная невырожденная гамильтонова структура гидродинамического типа (скобка Пуассона типа Дубровина-Новикова [5]). В отличие от (1.3) интегрируемая система гидродинамического типа (1.4) обладает только нелокальными структурами гидродинамического типа (см. [14-16]). Исследование, проведенное в $[2,3]$, показало, что системы (1.3) и (1.4) являются недиагонализуемыми (т.е. не допускают инвариантов Римана).

В $\S 3$ мы излагаем общую теорию интегрируемости недиагонализуемых гамильтоновых систем гидродинамического типа порядка $3 \times 3$, следуя [6-9]. Оказывается, что всякая такая система сводится некоторой стандартной цепочкой преобразований к интегрируемой системе трех волн. Мы демонстрируем работу этой процедуры на примере системы (1.3). Соответственно, показано, что любое решение интегрируемой системы трех волн порождает трехпараметрическое семейство решений уравнения ассоциативности (1.1).

В $\S 4$ строится явное преобразование типа Беклунда, связывающее решения систем (1.3) и (1.4).

\section{§2. Гамильтоново представление системы (1.3)}

Как было замечено Дубровиным [1], уравнение (1.1) связано со спектральной задачей, которая в переменных $a, b, c$ приобретает следующий вид:

$$
\Psi_{x}=z A \Psi=z\left(\begin{array}{ccc}
0 & 1 & 0 \\
b & a & 1 \\
c & b & 0
\end{array}\right) \Psi, \quad \Psi_{t}=z B \Psi=z\left(\begin{array}{ccc}
0 & 0 & 1 \\
c & b & 0 \\
b^{2}-a c & c & 0
\end{array}\right) \Psi .
$$

Условия совместности спектральной задачи (2.1) эквивалентны следующим двум соотношениям между матрицами $A$ и $B$ :

$$
\left\{\begin{array}{l}
A_{t}=B_{x} \\
{[A, B]=0,}
\end{array}\right.
$$

которые выполняются тождественно в силу уравнений $(1.3)$ (здесь [ , ] обозначает коммутатор).

Лемма 1. Собственные числа матрицы А лвляются плотностяли законов сохранения системь (1.3).

ДоказАТЕЛЬСтво. Поскольку матрицы $A$ и $B$ коммутируют и имеют простой спектр, они могут быть одновременно диагонализованы:

$$
A=P U P^{-1}, \quad B=P V P^{-1} .
$$

Здесь $U=\operatorname{diag}\left(u^{1}, u^{2}, u^{3}\right), V=\operatorname{diag}\left(v^{1}, v^{2}, v^{3}\right)$. Подстановка в уравнение $(2.2)$ дает

$$
\left[P^{-1} P_{t}, U\right]+U_{t}=\left[P^{-1} P_{x}, V\right]+V_{x} .
$$


Осталось заметить, что матрицы $\left[P^{-1} P_{t}, U\right]$ и $\left[P^{-1} P_{x}, V\right]$ являются внедиагональными. Следовательно,

$$
U_{t}=V_{x}
$$

Таким образом, помимо трех очевидных законов сохранения с плотностями $a, b, c$ система (1.3) имеет три закона сохранения с плотностями $u^{1}, u^{2}, u^{3}$, являющимися корнями характеристического уравнения

$$
\operatorname{det}(\lambda E-A)=\lambda^{3}-a \lambda^{2}-2 b \lambda-c=0 .
$$

В силу очевидного соотношения $a=u^{1}+u^{2}+u^{3}$ среди них лишь пять законов сохранения с плотностями $u^{1}, u^{2}, u^{3}, b, c$ являются линейно независимыми. Можно показать, что система (1.3) не имеет других законов сохранения гидродинамического типа, т.е. с плотностями вида $h(a, b, c)$.

Перейдем в уравнениях (1.3) от переменных $a, b, c$ к новым зависимым переменным $u^{1}, u^{2}, u^{3}$, связанным с $a, b, c$ формулами Виета

$$
a=u^{1}+u^{2}+u^{3}, \quad b=-\frac{1}{2}\left(u^{1} u^{2}+u^{2} u^{3}+u^{3} u^{1}\right), \quad c=u^{1} u^{2} u^{3} .
$$

Для сокращения необходимых вычислений заметим, что матрицы $A$ и $B$ связаны формулой

$$
B=A^{2}-a A-b E .
$$

Следовательно, то же самое соотношение имеет место и для соответствующих диагональных матриц $U$ и $V$ :

$$
V=U^{2}-a U-b E
$$

Подставляя выражения для $a$ и $b$ и используя уравнение $(2.2)$, получаем

$$
U_{t}=\left(U^{2}-\left(u^{1}+u^{2}+u^{3}\right) U+\frac{1}{2}\left(u^{1} u^{2}+u^{2} u^{3}+u^{3} u^{1}\right) E\right)_{x}
$$

или, в компонентах,

$$
\left(\begin{array}{l}
u^{1} \\
u^{2} \\
u^{3}
\end{array}\right)_{t}=\frac{1}{2}\left(\begin{array}{c}
u^{2} u^{3}-u^{1} u^{2}-u^{1} u^{3} \\
u^{1} u^{3}-u^{2} u^{1}-u^{2} u^{3} \\
u^{1} u^{2}-u^{3} u^{1}-u^{3} u^{2}
\end{array}\right)_{x}=\frac{1}{2}\left(\begin{array}{rrr}
1 & -1 & -1 \\
-1 & 1 & -1 \\
-1 & -1 & 1
\end{array}\right) \frac{d}{d x}\left(\begin{array}{l}
\partial h / \partial u^{1} \\
\partial h / \partial u^{2} \\
\partial h / \partial u^{3}
\end{array}\right),
$$

где $h=c=u^{1} u^{2} u^{3}$. Следовательно, рассматриваемая система является гамильтоновой с гамильтоновым оператором

$$
M=\frac{1}{2}\left(\begin{array}{rrr}
1 & -1 & -1 \\
-1 & 1 & -1 \\
-1 & -1 & 1
\end{array}\right) \frac{d}{d x}
$$

и гамильтонианом $H=\int c d x=\int u^{1} u^{2} u^{3} d x$. Плотностью импульса и аннуляторами соответствующей скобки Пуассона являются:

$$
\begin{aligned}
& 2 b=-u^{1} u^{2}-u^{2} u^{3}-u^{3} u^{1} \text { (плотность импульса), } \\
& u^{1}, u^{2}, u^{3} \text { (аннуляторы). }
\end{aligned}
$$


В исходных переменных $a, b, c$ гамильтонов оператор (2.4) имеет вид

$$
M=\left(\begin{array}{ccc}
-\frac{3}{2} & \frac{1}{2} a & b \\
\frac{1}{2} a & b & \frac{3}{2} c \\
b & \frac{3}{2} c & 2\left(b^{2}-a c\right)
\end{array}\right) \frac{d}{d x}+\left(\begin{array}{ccc}
0 & \frac{1}{2} a_{x} & b_{x} \\
0 & \frac{1}{2} b_{x} & c_{x} \\
0 & \frac{1}{2} c_{x} & \left(b^{2}-a c\right)_{x}
\end{array}\right)
$$

Напомним, что локальные невырожденные гамильтоновы операторы гидродинамического типа были введены и изучены Дубровиным и Новиковым (см. [5]). Они доказали, что оператор вида

$$
P^{i j}=g^{i j}(u) \frac{d}{d x}+b_{k}^{i j}(u) u_{x}^{k}, \quad \operatorname{det}\left[g^{i j}(u)\right] \neq 0,
$$

гамильтонов тогда и только тогда, когда

(1) $g^{i j}(u)$ - метрика нулевой римановой кривизны (т. е. плоская метрика);

(2) $b_{k}^{i j}(u)=-g^{i s}(u) \Gamma_{s k}^{j}(u)$, где $\Gamma_{s k}^{j}(u)$ - коэффициенты дифференциальногеометрической связности, порождаемой метрикой $g^{i j}$, т. е. симметричной связности, согласованной с метрикой (связности Леви-Чивиты).

Гамильтоновы системы гидродинамического типа, введенные Дубровиным и Новиковым (см. [5]), имеют вид

$$
u_{t}^{i}=P^{i j} \frac{\partial H}{\partial u^{j}},
$$

где $H=\int h(u) d x-$ функционал гидродинамического типа. Нелокальные обобщения гамильтоновой теории систем гидродинамического типа были открыты в [14] (см. также $[15,16])$. В работах Царева [4] была построена эффективная теория интегрируемости диагонализуемых гамильтоновых систем гидродинамического типа, т. е. гамильтоновых систем, приводимых к инвариантам Римана:

$$
R_{t}^{i}=v^{i}(R) R_{x}^{i} .
$$

Все такие системы допускают бесконечный набор законов сохранения и коммутирующих потоков гидродинамического типа и интегрируются обобщенным методом годографа. Однако в работах $[2,3]$ было показано, что система $(1.3)$ не допускает инвариантов Римана. Это объясняет, в частности, то обстоятельство, что система (1.3) имеет лишь конечное число гидродинамических интегралов.

Общая теория интегрируемости недиагонализуемых (т. е. не допускающих инвариантов Римана) гамильтоновых систем гидродинамического типа развивалась в [6-9]. Для трехкомпонентных систем полученные результаты носят окончательный характер.

ТеОРема $1[7,8]$. Недиагонализуемая гамильтонова (с невырожденной скобкой Пуассона) система гидродинамического типа порлдка $3 \times 3$ является интегрируемой тогда и только тогда, когда она слабо нелинейна.

Напомним, что система гидродинамического типа

$$
u_{t}^{i}=v_{j}^{i}(u) u_{x}^{j}, \quad i, j=1, \ldots, n,
$$

называется слабо нелинейной, если для собственных чисел $\lambda^{i}(u)$ матрицы $v_{j}^{i}(u)$ при любом $i=1, \ldots, n$ выполнены соотношения

$$
L_{\vec{X}^{i}}\left(\lambda^{i}\right)=0,
$$


где $L_{\vec{X}^{i}}$ - производная Ли вдоль собственного вектора $\vec{X}^{i}$, отвечающего собственному значению $\lambda^{i}$.

Имеется простой и эффективный критерий слабой нелинейности, не апеллирующий к собственным числам и собственным векторам.

ПРЕДЛОЖЕНИЕ [7]. Система гидродинамического типа (2.5) является слабо нелинейной тогда и только тогда, когда

$$
\left(\operatorname{grad} f_{1}\right) v^{n-1}+\left(\operatorname{grad} f_{2}\right) v^{n-2}+\cdots+\left(\operatorname{grad} f_{n}\right) E=0,
$$

где $f_{i}$ - коэффициенты характеристического многочлена

$$
\operatorname{det}\left(\lambda \delta_{j}^{i}-v_{j}^{i}(u)\right)=\lambda^{n}+f_{1}(u) \lambda^{n-1}+f_{2}(u) \lambda^{n-2}+\cdots+f_{n}(u),
$$

a $v^{n}$ обозначает $n$-ю степень матриць $v_{j}^{i}$.

Как было показано в [2, 3], система (1.3) является слабо нелинейной.

\section{§3. Интегрируемые гамильтоновы системы гидродинамического типа порядка $3 \times 3$, не приводимые к инвариантам Римана}

Рассмотрим систему гидродинамического типа

$$
u_{t}^{i}=v_{j}^{i}(u) u_{x}^{j} .
$$

Пусть $\lambda^{i}(u)$ - собственные числа матрицы $v_{j}^{i}$, т. е. корни характеристического уравнения $\operatorname{det}\left(v_{j}^{i}(u)-\lambda \delta_{j}^{i}\right)=0$ (мы предполагаем, что рассматриваемая система является строго гиперболической, т.е. все корни характеристического уравнения вещественны и различны). Обозначим через $\vec{l}^{i}(u)=\left(l_{1}^{i}, \ldots, l_{n}^{i}\right)$ левый собственный вектор матрицы $v_{j}^{i}$, отвечающий собственному значению $\lambda^{i}$, т.е. $l_{k}^{i} v_{j}^{k}=\lambda^{i} l_{j}^{i}$. Введем в рассмотрение 1-формы $\omega^{i}=l_{k}^{i} d u^{k}(i=1, \ldots, n)$. Подчеркнем, что 1-формы $\omega^{i}$ определены с точностью до нормировки $\omega^{i} \mapsto p^{i} \omega^{i}$, $p^{i} \neq 0$. Несложно убедиться в том, что уравнения (3.1) могут быть переписаны в виде системы внешних уравнений

$$
\omega^{i} \wedge\left(d x+\lambda^{i} d t\right)=0, \quad i=1, \ldots, n .
$$

Для рассматриваемой нами системы (2.3) собственные числа $\lambda^{i}$ и отвечающие им левые собственные векторы $\vec{l}^{i}$ имеют вид

$$
\begin{array}{ll}
\lambda^{1}=-u^{1}, & \vec{l}^{1}=\left(u^{2}-u^{3}, u^{1}-u^{3}, u^{2}-u^{1}\right), \\
\lambda^{2}=-u^{2}, & \vec{l}^{2}=\left(u^{2}-u^{3}, u^{1}-u^{3}, u^{1}-u^{2}\right), \\
\lambda^{3}=-u^{3}, & \vec{l}^{3}=\left(u^{2}-u^{3}, u^{3}-u^{1}, u^{2}-u^{1}\right) .
\end{array}
$$

Следовательно, уравнения (2.3) могут быть переписаны в виде

$$
\omega^{i} \wedge\left(d x-u^{i} d t\right)=0, \quad i=1,2,3,
$$


где

$$
\begin{aligned}
& \omega^{1}=\left(u^{2}-u^{3}\right) d u^{1}+\left(u^{1}-u^{3}\right) d u^{2}+\left(u^{2}-u^{1}\right) d u^{3}, \\
& \omega^{2}=\left(u^{2}-u^{3}\right) d u^{1}+\left(u^{1}-u^{3}\right) d u^{2}+\left(u^{1}-u^{2}\right) d u^{3}, \\
& \omega^{3}=\left(u^{2}-u^{3}\right) d u^{1}+\left(u^{3}-u^{1}\right) d u^{2}+\left(u^{2}-u^{1}\right) d u^{3} .
\end{aligned}
$$

Пусть $B(u) d x+A(u) d t$ и $N(u) d x+M(u) d t$ - два интеграла гидродинамического типа системы (3.1), т. е. дифференциальные формы замкнуты вдоль решений этой системы. Перейдем от переменных $x, t$ к новым независимым переменным $\tilde{x}, \tilde{t}$ по формулам

$$
d \tilde{x}=B d x+A d t, \quad d \tilde{t}=N d x+M d t .
$$

При этом система (3.1) преобразуется к виду

$$
u_{\tilde{t}}^{i}=\tilde{v}_{j}^{i}(u) u_{\tilde{x}}^{j},
$$

где матрица $\tilde{v}$ связана с $v$ формулой

$$
\tilde{v}=(B v-A E)(M E-N v)^{-1} .
$$

На языке внешних уравнений преобразованная система запишется в виде

$$
\omega^{i} \wedge\left(d \tilde{x}+\tilde{\lambda}^{i} d \tilde{t}\right)=0, \quad i=1, \ldots, n,
$$

где

$$
\tilde{\lambda}^{i}=\frac{\lambda^{i} B-A}{M-\lambda^{i} N} .
$$

Следовательно, при преобразованиях вида (3.4) 1-формы $\omega^{i}$ не меняются, в то время как собственные числа $\lambda^{i}$ преобразуются по формуле (3.6).

Теорема 2 [7, 8]. Пусть система гидродинамического типа (3.1) порядка $3 \times 3$ является слабо нелинейной и гамильтоновой (с невырожденной скобкой Пуассона гидродинамического типа). Тогда у нее найдется такая пара интегралов (3.4), что преобразованная система будет иметь постоянные собственные числа $\tilde{\lambda}^{i}$, которые без ограничения общности можно считать такими: $\tilde{\lambda}^{1}=1, \tilde{\lambda}^{2}=-1, \tilde{\lambda}^{3}=0$.

Для системы (2.3) преобразование (3.4), существование которого утверждается теоремой 2, имеет следующий вид:

$$
\begin{gathered}
d \tilde{x}=B d x+A d t=\left(u^{1}-u^{2}\right) d x+u^{3}\left(u^{2}-u^{1}\right) d t \\
d \tilde{t}=N d x+M d t=\left(2 u^{3}-u^{1}-u^{2}\right) d x+\left(2 u^{1} u^{2}-u^{1} u^{3}-u^{2} u^{3}\right) d t .
\end{gathered}
$$

Согласно формуле (3.6), преобразованные собственные числа будут равны 1 , $-1,0$ соответственно. Следовательно, в новых независимых переменных $\tilde{x}, \tilde{t}$ система (2.3) перепишется в виде

$$
\omega^{1} \wedge(d \tilde{x}+d \tilde{t})=0, \quad \omega^{2} \wedge(d \tilde{x}-d \tilde{t})=0, \quad \omega^{3} \wedge d \tilde{x}=0 .
$$


Теорема $3[7,8]$. Пусть система гидродинамического типа (3.1) порядка $3 \times 3$ является недиагонализуемой, слабо нелинейной и гамильтоновой ( $c$ невырожденной скобкой Пуассона гидродинамического типа). Тогда соответствуюшие 1-формы $\omega^{1}, \omega^{2}, \omega^{3}$ могут быть нормированы таким образом, что для них будут выполнены либо структурные уравнения группы $S O(3)$

$$
d \omega^{1}=\omega^{2} \wedge \omega^{3}, \quad d \omega^{2}=\omega^{3} \wedge \omega^{1}, \quad d \omega^{3}=\omega^{1} \wedge \omega^{2},
$$

если сигнатура метрики, задающей скобку Пуассона, лвляется евклидовой, либо структурнье уравнения группь $S O(2,1)$

$$
d \omega^{1}=\omega^{2} \wedge \omega^{3}, \quad d \omega^{2}=\omega^{3} \wedge \omega^{1}, \quad d \omega^{3}=-\omega^{1} \wedge \omega^{2}
$$

(в случае лоренцевой сигнатурь).

Для системы (2.3) сигнатура метрики скобки Пуассона (2.4) является лоренцевой. Следовательно, формы (3.3) могут быть нормированы таким образом, что для них будут выполнены структурные уравнения (3.9). Искомая нормировка имеет вид (мы не будем вводить переобозначений для нормированных $\left.1-ф о р м ~ \omega^{i}\right)$ :

$$
\begin{aligned}
& \omega^{1}=\frac{\left(u^{2}-u^{3}\right) d u^{1}+\left(u^{1}-u^{3}\right) d u^{2}+\left(u^{2}-u^{1}\right) d u^{3}}{2\left(u^{2}-u^{3}\right) \sqrt{\left(u^{2}-u^{1}\right)\left(u^{3}-u^{1}\right)}}, \\
& \omega^{2}=\frac{\left(u^{2}-u^{3}\right) d u^{1}+\left(u^{1}-u^{3}\right) d u^{2}+\left(u^{1}-u^{2}\right) d u^{3}}{2\left(u^{3}-u^{1}\right) \sqrt{\left(u^{2}-u^{1}\right)\left(u^{2}-u^{3}\right)}}, \\
& \omega^{3}=\frac{\left(u^{2}-u^{3}\right) d u^{1}+\left(u^{3}-u^{1}\right) d u^{2}+\left(u^{2}-u^{1}\right) d u^{3}}{2\left(u^{2}-u^{1}\right) \sqrt{\left(u^{3}-u^{1}\right)\left(u^{2}-u^{3}\right)}}
\end{aligned}
$$

(для определенности мы считаем, что $u^{1}<u^{3}<u^{2}$ ).

Непосредственной проверкой можно убедиться, что 1-формы (3.10) удовлетворяют структурным уравнениям (3.9).

Итак, согласно теоремам 2 и 3 , всякая недиагонализуемая слабо нелинейная гамильтонова (с невырожденной скобкой Пуассона) система гидродинамического типа порядка $3 \times 3$ может быть приведена к каноническому виду

$$
\omega^{1} \wedge(d \tilde{x}+d \tilde{t})=0, \quad \omega^{2} \wedge(d \tilde{x}-d \tilde{t})=0, \quad \omega^{3} \wedge d \tilde{x}=0
$$

подходящим преобразованием (3.4). При этом можно считать, что для форм $\omega^{i}$ выполнены структурные уравнения (3.8) или (3.9) (заметим, что преобразования типа (3.4) не меняют структурных уравнений). Вводя в уравнениях (3.11) переменные $p^{1}, p^{2}, p^{3}$ по формулам (см. $\left.[6,7]\right)$

$$
\omega^{1}=p^{1}(d \tilde{x}+d \tilde{t}), \quad \omega^{2}=p^{2}(d \tilde{x}-d \tilde{t}), \quad \omega^{3}=p^{3} d \tilde{x}
$$

и подставляя (3.12) в структурные уравнения (для определенности в (3.9)), мы получим интегрируемую систему трех волн

$$
p_{\tilde{t}}^{1}-p_{\tilde{x}}^{1}=-p^{2} p^{3}, \quad p_{\tilde{t}}^{2}+p_{\tilde{x}}^{2}=-p^{1} p^{3}, \quad p_{\tilde{t}}^{3}=-2 p^{1} p^{2} .
$$

ЗАмЕЧАНИЕ. Если воспользоваться явной координатной записью 1-форм $\omega^{i}=$ $l_{k}^{i}(u) d u^{k}$, то для $p^{i}$ получатся выражения вида $p^{i}=l_{k}^{i}(u) u_{\tilde{x}}^{k}$. Следовательно, переход от $u^{i}$ к $p^{i}$ является дифференциальной подстановкой первого порядка. 
Подытоживая описанную конструкцию применительно к рассматриваемой нами системе, мы можем условно разбить переход от уравнений (2.3) к системе трех волн (3.13) на два этапа:

1. Переход от $x, t$ к новым независимым переменным $\tilde{x}, \tilde{t}$ по формулам (3.7).

2. Переход от переменных $u^{1}, u^{2}, u^{3}$ к $p^{1}, p^{2}, p^{3}$ по формулам (ср. с (3.10))

$$
\begin{aligned}
& p^{1}=\frac{\left(u^{2}-u^{3}\right) u_{\tilde{x}}^{1}+\left(u^{1}-u^{3}\right) u_{\tilde{x}}^{2}+\left(u^{2}-u^{1}\right) u_{\tilde{x}}^{3}}{2\left(u^{2}-u^{3}\right) \sqrt{\left(u^{2}-u^{1}\right)\left(u^{3}-u^{1}\right)}}, \\
& p^{2}=\frac{\left(u^{2}-u^{3}\right) u_{\tilde{x}}^{1}+\left(u^{1}-u^{3}\right) u_{\tilde{x}}^{2}+\left(u^{1}-u^{2}\right) u_{\tilde{x}}^{3}}{2\left(u^{3}-u^{1}\right) \sqrt{\left(u^{2}-u^{1}\right)\left(u^{2}-u^{3}\right)}}, \\
& p^{3}=\frac{\left(u^{2}-u^{3}\right) u_{\tilde{x}}^{1}+\left(u^{3}-u^{1}\right) u_{\tilde{x}}^{2}+\left(u^{2}-u^{1}\right) u_{\tilde{x}}^{3}}{2\left(u^{2}-u^{1}\right) \sqrt{\left(u^{3}-u^{1}\right)\left(u^{2}-u^{3}\right)}} .
\end{aligned}
$$

Таким образом, всякое решение интегрируемой системы трех волн (3.13) порождает трехпараметрическое семейство решений уравнения ассоциативности (1.1).

\section{§4. Связь систем (1.3) и (1.4)}

Спектральная задача, отвечающая системе (1.4), имеет вид

$$
\begin{aligned}
& \Psi_{x}=z A \Psi=z\left(\begin{array}{lll}
0 & 1 & 0 \\
0 & b & a \\
1 & c & b
\end{array}\right) \Psi \\
& \Psi_{t}=z B \Psi=z\left(\begin{array}{ccc}
0 & 0 & 1 \\
1 & c & b \\
0 & (1+b c) / a & c
\end{array}\right) \Psi .
\end{aligned}
$$

Легко проверить, что матрица $B$ выражается через $A$ по формуле

$$
B=\frac{1}{a}\left(A^{2}-b A\right) \text {. }
$$

Условие совместности спектральной задачи (4.1)

$$
A_{t}=B_{x}
$$

переписанное в терминах собственных чисел матриц $A$ и $B$ (см. лемму 1 из $\S 2$ ), имеет вид

$$
w_{t}^{i}=\left(\left(\left(w^{i}\right)^{2}-w^{i} b\right) / a\right)_{x},
$$

где $w^{i}$ - собственные числа матрицы $A$, т. е. корни характеристического уравнения

$$
\operatorname{det}(\lambda E-A)=\lambda^{3}-2 b \lambda^{2}+\left(b^{2}-a c\right) \lambda-a=0 .
$$

Выражая отсюда $a$ и $b$ по формулам Виета,

$$
b=\frac{1}{2}\left(w^{1}+w^{2}+w^{3}\right), \quad a=w^{1} w^{2} w^{3},
$$


и подставляя полученные выражения в (4.2), мы получим явную запись уравнений (1.4) в координатах $w^{i}$ :

$$
\left(\begin{array}{c}
w^{1} \\
w^{2} \\
w^{3}
\end{array}\right)_{t}=\frac{1}{2}\left(\begin{array}{c}
\left(w^{1}-w^{2}-w^{3}\right) / w^{2} w^{3} \\
\left(w^{2}-w^{1}-w^{3}\right) / w^{1} w^{3} \\
\left(w^{3}-w^{1}-w^{2}\right) / w^{1} w^{2}
\end{array}\right)_{x} .
$$

Отметим, что интегрируемые системы гидродинамического типа (1.4) и (4.3) не обладают локальными гамильтоновыми структурами гидродинамического типа (скобками Пуассона типа Дубровина-Новикова [5]), - отвечающие им гамильтоновы структуры гидродинамического типа нелокальны (см. [14-16]).

Укажем явную связь между системами (2.3) и (4.3). Для этого перейдем в уравнениях (2.3) от $x, t$ к новым независимым переменным $\tilde{x}, \tilde{t}$ по формулам

$$
d \tilde{x}=-\frac{1}{2}\left(u^{1} u^{2}+u^{1} u^{3}+u^{2} u^{3}\right) d x+u^{1} u^{2} u^{3} d t, \quad d \tilde{t}=d x .
$$

После преобразования (4.4) система (2.3) принимает вид

$$
\left(\begin{array}{c}
1 / u^{1} \\
1 / u^{2} \\
1 / u^{3}
\end{array}\right)_{\tilde{t}}=\frac{1}{2}\left(\begin{array}{c}
\left(u^{2} u^{3}\right) / u^{1}-u^{2}-u^{3} \\
\left(u^{1} u^{3}\right) / u^{2}-u^{1}-u^{3} \\
\left(u^{1} u^{2}\right) / u^{3}-u^{1}-u^{2}
\end{array}\right)_{\tilde{x}}
$$

что, как легко видеть, совпадает с (4.3) после преобразования

$$
w^{i}=1 / u^{i} \text {. }
$$

На языке исходных уравнений (1.1) и (1.2) преобразования (4.4) и (4.5) могут быть представлены следующим образом: уравнение

$$
f_{t t t}=f_{x x t}^{2}-f_{x x x} f_{x t t}
$$

переходит в уравнение

$$
\tilde{f}_{\tilde{x} \tilde{x} \tilde{x}} \tilde{f}_{\tilde{t} \tilde{t} \tilde{t}}-\tilde{f}_{\tilde{x} \tilde{x} \tilde{t}} \tilde{f}_{\tilde{x} \tilde{t} \tilde{t}}=1
$$

при замене

$$
\tilde{x}=f_{x t}, \quad \tilde{t}=x, \quad \tilde{f}_{\tilde{x} \tilde{x}}=t, \quad \tilde{f}_{\tilde{x} \tilde{t}}=-f_{x x}, \quad \tilde{f}_{\tilde{t} \tilde{t}}=f_{t t} .
$$

Отметим, что это преобразование, связывающее решения уравнений ассоциативности (1.1) и (1.2), напоминает по своему виду автопреобразования уравнений ассоциативности, найденные Дубровиным (преобразования типа Лежандра [1]), но, в отличие от них, не сохраняет метрику $\eta_{\alpha \beta}$.

Авторы благодарят Б. А. Дубровина, привлекшего их внимание к уравнениям ассоциативности, а также И. М. Кричевера и С. П. Новикова за полезные обсуждения.

\section{ЛИТЕРАТУРА}

1. Dubrovin B. Geometry of $2 \mathrm{D}$ topological field theories. Preprint SISSA-89/94/FM, SISSA, Trieste (1994).

2. Mokhov O. I. Differential equations of associativity in $2 \mathrm{D}$ topological field theories and geometry of nondiagonalizable systems of hydrodynamic type. In: Abstracts 
of Internat. Conference on Integrable Systems "Nonlinearity and Integrability: from Mathematics to Physics," February 21-24, 1995, Montpellier, France (1995).

3. Mokhov O. I. Symplectic and Poisson geometry on loop spaces of manifolds and nonlinear equations. In: Topics in topology and mathematical physics (S. P. Novikov ed.), Transl. Am. Math. Soc., Ser. 2, Vol. 170, Am. Math. Soc., Providence, 121-151 (1995); hep-th/9503076.

4. Царев С. П. Геометрия гамильтоновых систем гидродинамического типа. Обобщенный метод годографа. Изв. АН СССР, Сер. матем., 54, вып. 5, 1048-1068 (1990).

5. Дубровин Б. А., Новиков С. П. Гидродинамика слабо деформированных солитонных решеток. Дифференциальная геометрия и гамильтонова теория. УМН, 44, вып. 6, 29-98 (1989).

6. Ferapontov E. V. On integrability of $3 \times 3$ semi-Hamiltonian hydrodynamic type systems which do not possess Riemann invariants. Phys. D, 63, 50-70 (1993).

7. Ferapontov E. $V$. On the matrix Hopf equation and integrable Hamiltonian systems of hydrodynamic type, which do not possess Riemann invariants. Phys. Lett. A, 179, 391-397 (1993).

8. Ferapontov E. V. Dupin hypersurfaces and integrable Hamiltonian systems of hydrodynamic type which do not possess Riemann invariants. Diff. Geom. Appl., 5, 121-152 (1995).

9. Ferapontov E. V. Several conjectures and results in the theory of integrable Hamiltonian systems of hydrodynamic type, which do not possess Riemann invariants. Теор. мат. физ., 99, вып. 2, 257-262 (1994).

10. Witten $E$. On the structure of the topological phase of two-dimensional gravity. Nuclear Phys. B, 340, 281-332 (1990).

11. Dijkgraaf R., Verlinde H., Verlinde E. Topological strings in $d<1$. Nuclear Phys. B, 352, 59-86 (1991).

12. Witten E. Two-dimensional gravity and intersection theory on moduli space. Surveys in Diff. Geom., 1, 243-310 (1991).

13. Dubrovin B. Integrable systems in topological field theory. Nuclear Phys. B, 379, 627-689 (1992).

14. Мохов О. И., Ферапонтов Е. В. О нелокальных гамильтоновых операторах гидродинамического типа, связанных с метриками постоянной кривизны. УМН, 45, вып. 3, 191-192 (1990).

15. Ферапонтов E. B. Дифференциальная геометрия нелокальных гамильтоновых операторов гидродинамического типа. Функц. анализ и его прил., 25, вып. 3, 37-49 (1991).

16. Mokhov O. I. Hamiltonian systems of hydrodynamic type and constant curvature metrics. Phys. Lett. A, 166, 215-216 (1992).

17. Mokhov O. I., Nutku Y. Bianchi transformation between the real hyperbolic MongeAmpère equation and the Born-Infeld equation. Lett. Math. Phys., 32, No. 2, 121-123 (1994).

Математический институт им. В. А. Стеклова РАН

Поступило в редакцию

Институт математического моделирования РАН

15 мая 1995 г. 